\title{
ON THE BANACH-MAZUR DISTANCE BETWEEN THE CUBE AND THE CROSSPOLYTOPE
}

\author{
FEI XUE
}

Abstract. In this note we study the Banach-Mazur distance between the $n$-dimensional cube and the crosspolytope. Previous work shows that the distance has order $\sqrt{n}$, and here we will prove some explicit bounds improving on former results. Even in dimension 3 the exact distance is not known, and based on computational results it is conjectured to be $\frac{9}{5}$. Here we will also present computer based potentially optimal results in dimension 4 to 8 .

Mathematics subject classification (2010): 52A20, 52A21, 52A40.

Keywords and phrases: Banach-Mazur distance, cube, crosspolytope, Hadamard matrix.

\section{REFERENCES}

[1] J. Bourgain And S. J. SZAREK, The Banach-Mazur distance to the cube and the Dvoretzky-Rogers factorization, Israel J. Math. 62 (1988), 169.

[2] A. A. Giannopoulos, A Note on the Banach-Mazur Distance to the Cube, Geometric Aspects of Functional Analysis., Operator Theory Advances and Applications 77 (1995), 67-73.

[3] E. D. GLusKin, The diameter of the Minkowski compactum is roughly equal to $n$ (Russian), Funktsional. Anal. i Prilozhen. 15, 1 (1981), 72-73.

[4] P. M. Gruber And C. G. LeKKeRKerker, Geometry of Numbers, North-Holland, Amsterdam, Netherlands, 1987.

[5] F. John, Extremum problems with inequalities as subsidiary conditions, Studies and Essays Presented to R. Courant on his 60th Birthday, Interscience Publishers, 1948, 187-204.

[6] J. J. SYLVESTER, Thoughts on inverse orthogonal matrices, simultaneous sign successions, and tessellated pavements in two or more colours, with applications to Newton's rule, ornamental tile-work, and the theory of numbers, Philosophical Magazine 34 (1867), 461-475.

[7] N. TOMCZAK-JAEGERMANN, Banach-Mazur distances and finite-dimensional operator ideals, Pitman monographs and surveys in pure and applied Mathematics, 38, 1989.

[8] R. VERSHYNIN, Lectures in geometric functional analysis, 2009, http://www-personal.umich.edu/ romanv/papers/GFA-book/GFA-book.pdf. 